\title{
Ultrasensitive determination for picomolar-level midecamycin in human serum by flow injection chemiluminescence using luminol-BSA system
}

\author{
Hairu Lv ${ }^{\mathrm{a}, \mathrm{b}}$, Xijuan Tan ${ }^{\mathrm{a}}$, Yun Zhang ${ }^{\mathrm{a}}$ and Zhenghua Song ${ }^{\mathrm{a}, *}$ \\ ${ }^{a}$ Key Laboratory of Synthetic and Natural Functional Molecule Chemistry of Ministry of Education, \\ College of Chemistry and Material Science, Northwest University, Xi'an, China \\ ${ }^{\mathrm{b}}$ Material Evidence Identifying Center of Xi'an Public Security Bureau, Xi'an, China
}

\begin{abstract}
An ultrasensitive method for determining picomolar midecamycin (MID) by flow injection (FI) chemiluminescence (CL) was first described based on the inhibitory effect of MID on luminol-BSA reaction. It was found that the CL intensity decrements were linear with the logarithm of MID concentrations in the range of $1.0-5000 \mathrm{pmol} \cdot 1^{-1}$ with a detection limit as low as $0.3 \mathrm{pmol} \cdot 1^{-1}(3 \sigma)$. The relative standard deviation of seven repetitive measurements for $10 \mathrm{pmol} \cdot 1^{-1} \mathrm{MID}$ was $3.0 \%$. At a flow rate of $2.0 \mathrm{ml} \cdot \mathrm{min}^{-1}$, the whole analysis procedure including sampling and washing could be finished in $30 \mathrm{~s}$, offering the sample efficiency of $120 \mathrm{~h}^{-1}$. This proposed method was successfully applied to determine MID in human serum samples with the recoveries from 96.0 to $110.0 \%$. The CL mechanism of luminol-BSA-MID reaction was also given.
\end{abstract}

Keywords: Midecamycin, BSA, luminol, chemiluminescence, flow injection

\section{Introduction}

Since flow injection (FI) analysis first described by Ruzicka and Hansen in 1975, it has revolutionized the performing way of analytical chemistry to a great extent [16]. Combined with chemiluminescence (CL), FI-CL, which possesses advantages of high sensitivity, low limit of detection (LOD), simple handling on-line or real-time monitoring, wide dynamic ranges, reproducibility and automatability as well as less reagent consumption, has become a very useful analytical tool in the science fields of biotechnology, pharmacology, food and environmental chemistry $[3,5,12,15]$. Luminol, one of the highest quantum yield luminescent compounds, has been extensively employed as the CL light emitting substrate in conjunction with different oxidants, such as metal ion [20,23], metal complex $[6,17]$, nanoparticle $[9,26]$,

\footnotetext{
${ }^{*}$ Corresponding author: Zhenghua Song, Key Laboratory of Synthetic and Natural Functional Molecule Chemistry of Ministry of Education, College of Chemistry and Material Science, Northwest University, Xi' an 710069, China. Tel.: +86 29 88303798; Fax: +86 29 88302604; E-mails: songzhenghua@hotmail.com; zhsong123@nwu.edu.cn.
} 


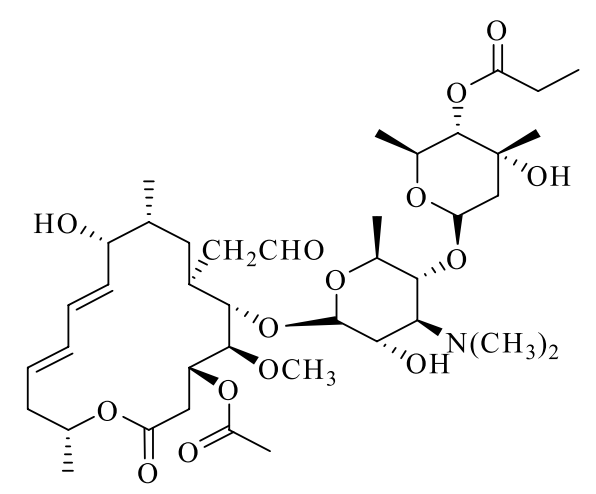

Fig. 1. The molecular structure of MID.

enzyme [4,24] and non-enzyme protein [19]. Recently, it has been reported that the hyperchromic effect of BSA on luminol can accelerate the electrons transferring rate of excited 3-aminophthalate leading to the CL intensity of luminol remarkably enhanced, and this FI-CL system has been developed successfully to assay azithromycin in pharmaceutical preparations [21]. So far there has been no report on the determination of midecamycin (MID) by FI-CL using luminol-BSA system.

MID $\left(\mathrm{C}_{41} \mathrm{H}_{67} \mathrm{NO}_{15}\right.$, Fig. 1), white crystalline powder with broad-spectrum against pneumococcal, streptococcal and mycoplasmal infections, is a widely used macrolide agent in the therapy of otitis media, urinary tract infection, skin soft tissue infection, and upper and lower respiratory tract infections $[1,2,7,13,14]$. Electrochemical analysis is the common-used method for the determination of MID, involving capillary zone electrophoresis (CZE) [10], cyclic voltammetry (CV) [8], adsorptive stripping voltammetry (ASV) [22], and polarographic catalytic wave (PCW) [18]. High-performance liquid chromatography with evaporative light-scattering detection (HPLC-ELSD) is also reported on determining MID [11]. In this present work, an ultrasensitive approach for determining MID by FI-CL was established for the first time on the basis of the inhibitory property of MID on luminol-BSA reaction. It was found that the CL intensity decrements were logarithm over MID concentrations, giving the calibration graph range of $1.0-5000 \mathrm{pmol} \cdot 1^{-1}$ with the LOD of $0.3 \mathrm{pmol} \cdot 1^{-1}(3 \sigma)$. This proposed method was successfully applied to determine MID in human serum samples with the recoveries from 96.0 to $110.0 \%$. The possible CL mechanism of luminol-BSA-MID reaction was also discussed in detail.

\section{Experimental section}

\subsection{Reagents}

All chemicals used in the whole experiments were of at least analytical reagent grade. Deionized water was purified in a Milli-Q system (Millipore, Bedford, MA, USA) before use. Luminol (Fluka, Biochemika) was purchased from Xi' an Medicine Purchasing and Supply Station, China. BSA (SigmaAldrich) was used directly without any further purification. MID was obtained from National Institute for the Control of Pharmaceutical and Biological Products, China.

MID stock standard solution $\left(2.0 \times 10^{-3} \mathrm{~g} \cdot \mathrm{ml}^{-1}\right)$ was prepared in ethanol-water solution $(1: 4, \mathrm{v} / \mathrm{v})$. The working standard solutions of MID were prepared daily from the stock solution as required. Luminol 
stock standard solution $\left(2.5 \times 10^{-2} \mathrm{~mol} \cdot \mathrm{1}^{-1}\right)$ was prepared in $1.0 \times 10^{-1} \mathrm{~mol} \cdot \mathrm{1}^{-1} \mathrm{NaOH}$ solution. BSA stock standard solution $\left(1.0 \times 10^{-6} \mathrm{~mol} \cdot \mathrm{l}^{-1}\right)$ was prepared with pure water and stored in a refrigerator at $4^{\circ} \mathrm{C}$.

\subsection{Apparatus}

The FI-CL system (Xi' an Remax Analysis Instrument Co. Ltd., Xi'an, China) was schematically illustrated in Fig. 2, including a sampling system (IFFM-E), a CL detector (IFFS-A) and a recorder (a computer with IFFM-E client system). The sample system provided a peristaltic pump to propel all solutions to the flow cell, and a six-way valve with loops of $100 \mu$ to inject quantitative luminol. The CL detector which was inside a black box to prevent ambient light contained a flow cell and a photomultiplier tube (PMT). The flow cell, which was placed close to the PMT, was made by coiling $15 \mathrm{~cm}$ length colorless glass tube $(1.0 \mathrm{~mm}$ i.d.) into a spiral disk shape $(2.0 \mathrm{~cm}$ i.d.). The $\mathrm{CL}$ signal from the flow cell was directly detected by the PMT without wavelength discrimination and the output was recorded on the computer. Polytetrafluoroethylene (PTFE) tubing (1.0 mm i.d.) was used to carry all the reagents.

\subsection{Procedures}

As shown in Fig. 2, all the streams (luminol, BSA, MID and carrier) were inserted into flow lines at a constant rate of $2.0 \mathrm{ml} \cdot \mathrm{min}^{-1}$ by the pump. The pure water was used to wash the whole flow system until a stable baseline had been recorded. Then $100 \mu \mathrm{l}$ of luminol was injected into the carrier stream by six-way valve and thereafter merged with the premixing stream of BSA and MID. The whole mixing solution was delivered to the CL cell in an alkaline medium and the CL intensity was detected by the PMT and luminosity meter. The concentrations of MID were measured by the CL intensity decrements $\left(\Delta I_{\mathrm{CL}}=I_{\mathrm{o}}-I_{\mathrm{s}}\right)$, where $I_{\mathrm{o}}$ and $I_{\mathrm{s}}$ were CL intensities in the absence and presence of MID, respectively.

\subsection{Treatment of spiked human serum samples}

The human serum samples from healthy volunteers were obtained from the Hospital of Northwest University. To prepare the spiked samples, known quantities of MID were added into $0.10 \mathrm{ml}$ human serum. After homogenization, suitable aliquot from the spiked samples with appropriate dilution were taken for the determination of MID.

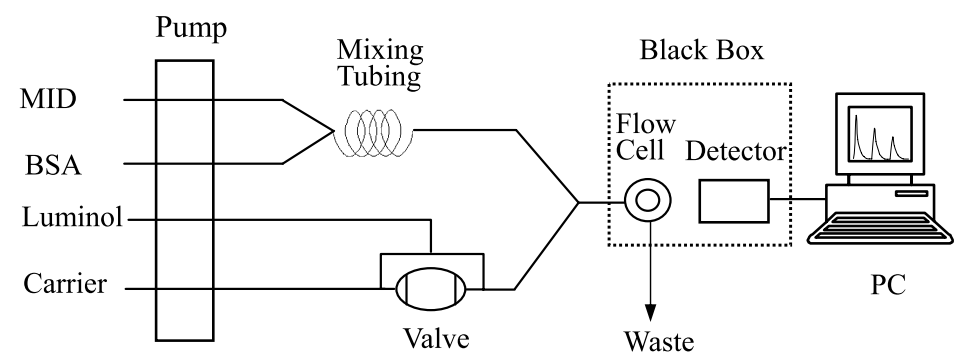

Fig. 2. Schematic diagram of the FI-CL system for the determination of MID. The concentrations of luminol, BSA and NaOH were $5.0 \times 10^{-5}, 5.0 \times 10^{-9}$ and $2.5 \times 10^{-2} \mathrm{~mol} \cdot 1^{-1}$, respectively. The flow rate was $2.0 \mathrm{ml} \cdot \mathrm{min}^{-1}$. The high voltage was $-750 \mathrm{~V}$. 


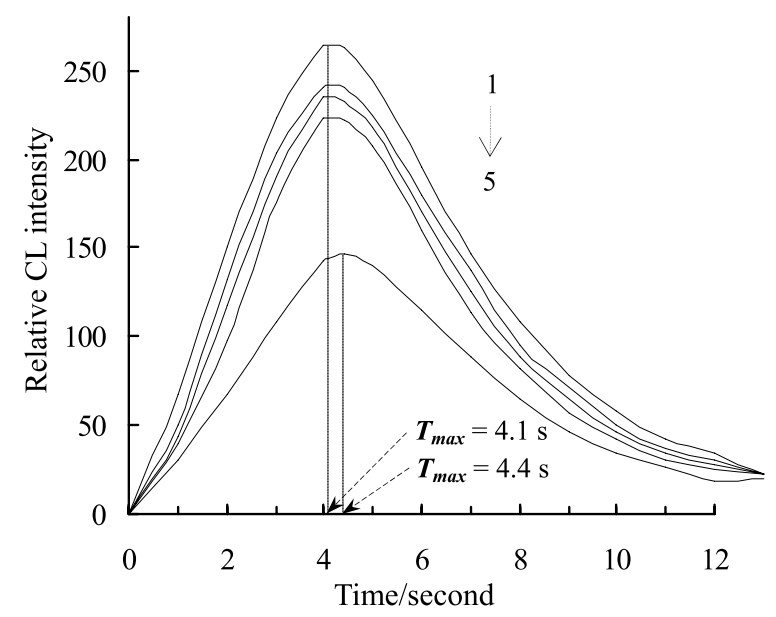

Fig. 3. Relative CL intensity-time profiles of different CL reactions: curve 1: luminol-BSA CL reaction; curves 2-4: luminol-BSA-MID CL reaction; curve 5: luminol-dissolved oxygen CL reaction. The concentrations of luminol and BSA were $5.0 \times 10^{-5}$ and $5.0 \times 10^{-9} \mathrm{~mol} \cdot 1^{-1}$. MID concentrations were $1.0 \times 10^{-12}, 1.0 \times 10^{-11}$ and $1.0 \times 10^{-9} \mathrm{~mol} \cdot \mathrm{l}^{-1}$, respectively.

\section{Results and discussion}

\subsection{The relative CL intensity-time profile}

The relative CL intensity-time profile was given in Fig. 3. It can be seen that the time $\left(T_{\max }\right)$ for reaching maximum CL intensity $\left(I_{\max }\right)$ of luminol-BSA reaction (curve 1) and luminol-dissolved oxygen reaction (curve 5) were 4.1 and 4.4 s with the $I_{\max }$ of 264 and 146, respectively; the $T_{\max }$ for luminol-BSA reaction in the presence of MID (curves 2-4) was $4.1 \mathrm{~s}$, while the $I_{\max }$ decreased proportionally to MID concentrations. The concentrations of luminol and BSA were $5.0 \times 10^{-5}$ and $5.0 \times 10^{-9} \mathrm{~mol} \cdot \mathrm{l}^{-1}$. MID concentrations from curves $2-4$ were $1.0 \times 10^{-12}, 1.0 \times 10^{-11}$ and $1.0 \times 10^{-9} \mathrm{~mol} \cdot 1^{-1}$, respectively.

\subsection{Optimum conditions of luminol-BSA CL system}

It was found that the concentrations of luminol and BSA greatly affected the CL intensity. To obtain a stable and strong CL intensity, $5.0 \times 10^{-5} \mathrm{~mol} \cdot 1^{-1}$ luminol and $5.0 \times 10^{-9} \mathrm{~mol} \cdot 1^{-1}$ BSA were selected in this work. Considering the nature of luminol CL reaction favoring under the alkaline medium, $2.5 \times 10^{-2} \mathrm{~mol} \cdot 1^{-1} \mathrm{NaOH}$ was added into the solution to enhance the CL intensity.

The effect of flow rate and mixing tube lengths was also examined. $2.0 \mathrm{ml} \cdot \mathrm{min}^{-1}$ flow rate and $5.0 \mathrm{~cm}$ mixing tube length were chosen in the subsequent experiments giving a strong CL intensity with a better precision, a good sensitivity and lower reagent consumption.

\subsection{Analytical performance for the determination of MID}

Under the optimized experimental conditions given above, the standard solutions of MID were determined. It was found that the CL intensity decrements were proportional to the logarithm of MID concentrations ranging from 1.0 to $5000 \mathrm{pmol} \cdot 1^{-1}$ with the LOD of $0.3 \mathrm{pmol} \cdot 1^{-1}(3 \sigma)$. The calibration equation for MID was $\Delta I_{\mathrm{CL}}=6.1 \lg C_{\mathrm{MID}}+22.8, \mathrm{R}^{2}=0.9921$. The relative standard deviations (RSDs) 
of seven replicate determinations of MID were 3.0, 2.4 and $1.7 \%$ at $10.0,100.0$ and $500.0 \mathrm{pmol} \cdot \mathrm{l}^{-1}$, respectively. One analyzing cycle of MID determination could be finished in $30 \mathrm{~s}$, which offered a sample efficiency of $120 \mathrm{~h}^{-1}$.

\subsection{Interference studies}

The interference studies were tested by adding potential interfering substances with increasing amounts into MID standard solution and the error was controlled under 5\%. The tolerable ratios of foreign substances with respect to $100 \mathrm{pmol} \cdot 1^{-1}$ MID were 100,000 for ethanol, glucose and fructose, 5000 for $\mathrm{NH}_{4}^{+}, \mathrm{Mg}^{2+}$ and $\mathrm{Ca}^{2+}, 1000$ for uric acid, 100 for $\mathrm{Fe}^{3+}$ and $\mathrm{Fe}^{2+}, 10$ for azithromycin. Under the optimum experimental conditions, abundant compounds such as protein, salt and lipid in human serum had no obvious interference for MID determination. Thus MID in human serum samples could be determined by FI-CL without separation step, which shortened the analyzing time and eliminated the errors from the separation procedure.

\subsection{Possible CL mechanism of luminol-BSA-MID reaction}

From Fig. 3, it was clear that the $I_{\max }$ of luminol-dissolved oxygen reaction in the presence of BSA (curve 1) increased from 146 to 264 and the $T_{\max }$ changed from 4.4 to $4.1 \mathrm{~s}$, showing BSA can accelerate the CL emission from luminol [21]; it was also clear that the $I_{\max }$ of luminol-BSA reaction was inhibited in the presence of MID (curves 2-4), however the $T_{\max }$ was the same as that of luminol-BSA reaction, indicating there may be interaction between BSA and MID. According to the FI-CL model of proteinsmall molecule interaction [25], the binding parameters of BSA with MID were obtained with $1.49 \times$ $10^{4} \mathrm{l} \cdot \mathrm{mol}^{-1}$ for the binding constant $K_{\mathrm{MID}}$ and 0.60 for the binding site number $n$. Hence, the possible CL mechanism of luminol-BSA-MID reaction can be explained as follows: the interaction between BSA and luminol with a 1:1 complex formed $\left(K_{\mathrm{L}}=1.12 \times 10^{7} \mathrm{l} \cdot \mathrm{mol}^{-1}\right)$ led to the CL intensity of luminol enhanced and produced the effect of complexing enhancement of CL [25]; while in the presence of MID, the BSA-MID complex $\left(K_{\mathrm{MID}}=1.49 \times 10^{4} 1 \cdot \mathrm{mol}^{-1}, n=0.60\right)$ formed online, which may cause the conformation of BSA changed and resulted in the CL intensity inhibition of luminol-BSA reaction.

\section{Application}

\subsection{Determination of MID in spiked human serum samples}

MID in the spiked human serum samples prepared in the experimental section were measured and the results were summarized in Table 1. It can be seen that the recoveries for the determination of MID were from 96.0 to $110.0 \%$ and the RSDs were less than $2.6 \%$, confirming the applicability of this method for MID determination in biological samples.

\section{Conclusion}

This paper described a sensitive method for the determination of MID by FI-CL with luminol-BSA system for the first time. Comparing with the reported methods for the measurement of MID shown in 
Table 1

The determination of MID in spiked human serum samples ${ }^{\mathrm{a}}$

\begin{tabular}{lcccc}
\hline $\begin{array}{l}\text { Sample } \\
\text { no. }\end{array}$ & $\begin{array}{c}\text { Added/Found } \\
\left(\mathrm{pg} \cdot \mathrm{ml}^{-1}\right)\end{array}$ & $\begin{array}{c}\text { RSD } \\
(\%)\end{array}$ & $\begin{array}{c}\text { Recovery } \\
(\%)\end{array}$ & $\begin{array}{c}\text { By proposed method/spiked } \\
\left(\mu \mathrm{g} \cdot \mathrm{ml}^{-1}\right)\end{array}$ \\
\hline 1 & $0 / 31$ & 2.2 & 96.7 & $0.62 / 0.60$ \\
& $30 / 60$ & 1.9 & & $0.58 / 0.60$ \\
2 & $0 / 29$ & 2.5 & 106.3 & $0.64 / 0.60$ \\
3 & $30 / 61$ & 2.0 & & $0.58 / 0.60$ \\
& $0 / 32$ & 2.3 & 96.0 & $0.94 / 1.00$ \\
4 & $50 / 80$ & 1.8 & & $1.02 / 1.00$ \\
5 & $0 / 29$ & 2.0 & 104.0 & \\
& $50 / 81$ & 1.3 & & $1.96 / 2.00$ \\
6 & $0 / 47$ & 2.6 & 110.0 & \\
& $30 / 80$ & 1.4 & & $2.02 / 2.00$ \\
7 & $0 / 51$ & 1.8 & 98.0 & \\
& $50 / 100$ & 1.5 & & \\
& $0 / 98$ & 1.6 & 103.0 & \\
& $100 / 203$ & 1.3 & & \\
& $0 / 101$ & 1.5 & 98.4 & \\
& $100 / 199$ & 1.4 & & \\
\hline
\end{tabular}

${ }^{\mathrm{a}}$ The average of five determinations.

Table 2

The comparison of FI-CL and other methods for determining MID

\begin{tabular}{lcccc}
\hline Methods & $\begin{array}{c}\text { Linear ranges } \\
\left(\mu \mathrm{mol} \cdot 1^{-1}\right)\end{array}$ & $\begin{array}{c}\text { LODs } \\
\left(\mathrm{nmol} \cdot 1^{-1}\right)\end{array}$ & Samples $^{\mathrm{a}}$ & Refs \\
\hline ASV & $2.5-6.1$ & $1.2 \times 10^{3}$ & Tablet, urine & {$[22]$} \\
CZE & $1.0-1000$ & $5.0 \times 10^{2}$ & Urine & {$[10]$} \\
HPLC-ELSD & $0.6-4900$ & - & Bulk material & {$[11]$} \\
CV & $0.5-20$ & - & Tablet & {$[8]$} \\
PCW & $2.0 \times 10^{-2}-1.0 \times 10$ & 6.0 & Tablet, urine & {$[18]$} \\
FI-CL & $1.0 \times 10^{-6}-5.0 \times 10^{-3}$ & $3.0 \times 10^{-4}$ & Serum & This study \\
\hline
\end{tabular}

${ }^{\mathrm{a}}$ The biological samples were from human beings.

Table 2, this proposed method exhibited excellent sensitivity with the LOD of $0.3 \mathrm{pmol} \cdot 1^{-1}$ which was about four orders in magnitude higher than others. The successful application of determining MID in human serum samples proved the ability of this method for analyzing complex biological matrix with separation-free, indicating its potential value in the pharmaceutical and clinic researches.

\section{Acknowledgements}

The authors gratefully acknowledge the financial support from Shaanxi Province Nature Science Foundation (No. 2006B05), the NWU Graduate Innovation and Creativity Funds (No. 09YZZ45) and the NWU Graduate Experimental Research Funds, China (Nos 09YSY18 and 10YZZ29). 


\section{References}

[1] E. Abu-Gharbieh, V. Vasina, E. Poluzzi and F. De Ponti, Antibacterial macrolides: a drug class with a complex pharmacological profile, Pharmacol. Res. 50 (2004), 211-222.

[2] S. Alvarez-Elcoro and J.D.C. Yao, Macrolide Antibiotics, 2nd edn, Elsevier, Amsterdam, 2003.

[3] D. Christodouleas, C. Fotakis, A. Economou, K. Papadopoulos, M. Timotheou-Potamia and A. Calokerinos, Flow-based methods with chemiluminescence detection for food and environmental analysis: a review, Anal. Lett. 44 (2011), 176-215.

[4] P.M. Easton, A.C. Simmonds, A. Rakishev, A.M. Egorov and L.P. Candeias, Quantitative model of the enhancement of peroxidase-induced luminol luminescence, J. Am. Chem. Soc. 118 (1996), 6619-6624.

[5] M. Guardigli, M. Mirasoli, E. Michelini, L.S. Dolci and A. Roda, Recent analytical application areas of chemiluminescence and bioluminescence, in: Chemiluminescence and Bioluminescence: Past, Present and Future, A. Roda, ed., RSC, Cambridge, 2010, pp. 557-573.

[6] T. Khajvand, M.J. Chaichi, O. Nazari and H. Golchoubian, Application of Box-Behnken design in the optimization of catalytic behavior of a new mixed chelate of copper (II) complex in chemiluminescence reaction of luminol, J. Lumin. 131 (2011), 838-842.

[7] H.A. Kirst, Antibiotics: macrolides, in: Van Nostrand's Encyclopedia of Chemistry, 5th edn, Wiley-Interscience, New York, 2005.

[8] N.B. Li, H.Q. Luo and G.N. Chen, Determination of medecamycin by adsorptive stripping voltammetry with an activated electrode, Anal. Bioanal. Chem. 38 (2004), 908-912.

[9] C. Liu and B.X. Li, Silver nanoparticle-initiated chemiluminescence reaction of luminol- $\mathrm{AgNO}_{3}$ and its analytical application, Anal. Bioanal. Chem. 401 (2011), 229-235.

[10] D.X. Liu and W.R. Jin, Determination of midecamycin by capillary zone electrophoresis with electrochemical detection, J. Chromatogr. B $\mathbf{7 8 3}$ (2003), 509-515.

[11] Y. Liu and C.Q. Hu, RP-HPLC determination of midecamycin and related impurities, Chromatographia 57 (2003), 143146.

[12] K. Mervartová, M. Polášek and J.M. Calatayud, Recent applications of flow-injection and sequential-injection analysis techniques to chemiluminescence determination of pharmaceuticals, J. Pharmaceut. Biomed. Anal. 45 (2007), 367-381.

[13] L. Meyler, Meyler's Side Effects of Drugs: The International Encyclopedia of Adverse Drug Reactions and Interactions, 15th edn, Elsevier, Amsterdam, 2006, p. 2343.

[14] J. Retsema and W.C. Fu, Macrolides: structures and microbial targets, Int. J. Antimicrob. Ag. 18 (2001), 3-10.

[15] A. Roda, P. Pasini, M. Mirasoli, E. Michelini and M. Guardigli, Biotechnological applications of bioluminescence and chemiluminescence, Trends Biotechnol. 22 (2004), 295-303.

[16] J. Ruzicka and E.H. Hansen, Flow injection analyses: Part I. A new concept of fast continuous flow analysis, Anal. Chim. Acta 78 (1975), 145-157.

[17] H.M. Shi, X.D. Xu, Y.X. Ding, S.P. Liu, L.Q. Li and W.J. Kang, Determination of cortisol in human blood sera by a new $\mathrm{Ag}(\mathrm{III})$ complex-luminol chemiluminescent system, Anal. Biochem. 387 (2009), 178-183.

[18] J.F. Song, N. Li and M.T. Xu, Study on association/parallel catalytic hydrogen wave in midecamycin-hydrogen peroxide system and its application, Acta Chim. Sinica 62 (2004), 78-82.

[19] Z.H. Song, L. Wang and S. Hou, A study of the chemiluminescence behavior of myoglobin with luminol and its analytical applications, Anal. Bioanal. Chem. 378 (2004), 529-535.

[20] T. Takayanagi, Y. Inaba, H. Kanzaki, Y. Jyoichi and S. Motomizu, Pre-evaluation of metal ions as a catalyst on chemiluminometric sequential injection analysis with luminol- $\mathrm{H}_{2} \mathrm{O}_{2}$ system, Talanta 79 (2009), 1089-1093.

[21] X.J. Tan, Z.H. Song, D.H. Chen and Z.M. Wang, Study on the chemiluminescence behavior of bovine serum albumin with luminol and its analytical application, Spectrochim. Acta A 79 (2011), 232-235.

[22] H.J. Wan, F.Q. Zhao and B.Z. Zeng, Direct electrochemistry and voltammetric determination of midecamycin at a multiwalled carbon nanotube coated gold electrode, Colloid. Surface B 86 (2011), 247-250.

[23] Z.H. Wang, F. Liu and C. Lu, Mg-Al-carbonate layered double hydroxides as a novel catalyst of luminol chemiluminescence, Chem. Commun. 47 (2011), 5479-5481.

[24] Z.M. Wang and Z.H. Song, A valuable way for understanding the relationships between lysozyme and cephalosporin analogues by flow injection chemiluminescence, Analyst 135 (2010), 2546-2553.

[25] Z.M. Wang, Z.H. Song and D.H. Chen, Study on the binding behavior of bovine serum albumin with cephalosporin analogues by chemiluminescence method, Talanta 83 (2010), 312-319.

[26] Z.F. Zhang, H. Cui, C.Z. Lai and L.J. Liu, Gold nanoparticle-catalyzed luminol chemiluminescence and its analytical applications, Anal. Chem. 77 (2005), 3324-3329. 


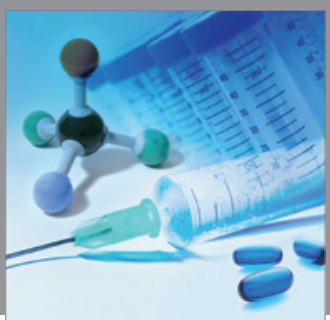

International Journal of

Medicinal Chemistry

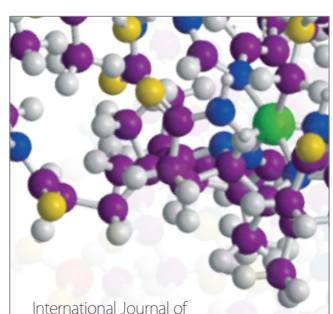

Carbohydrate Chemistry

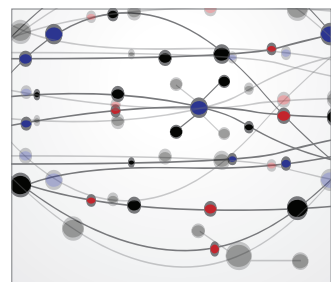

The Scientific World Journal
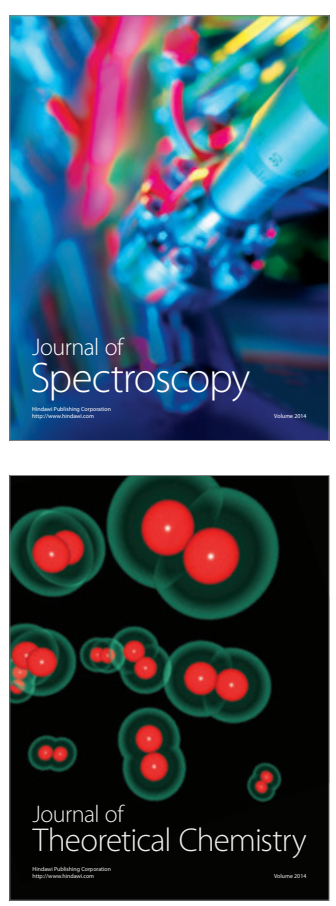
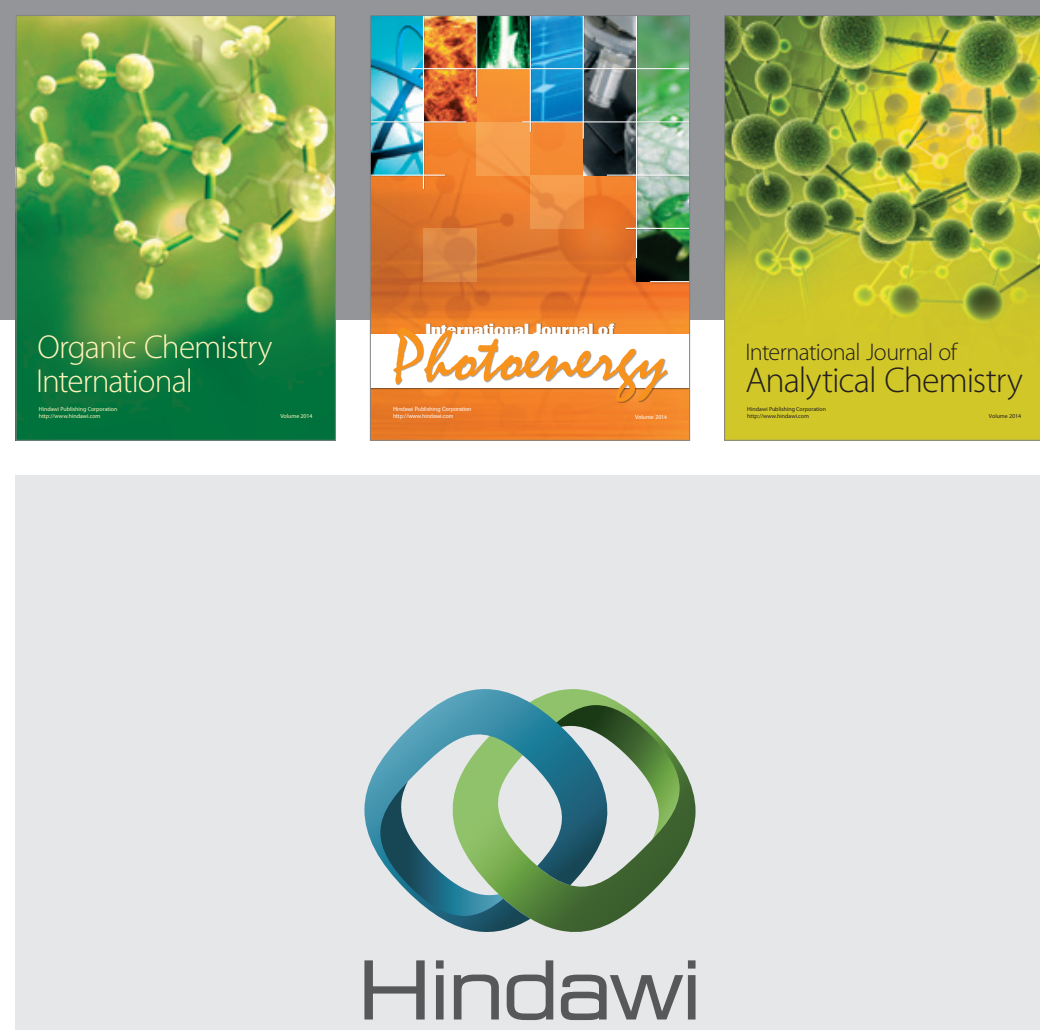

Submit your manuscripts at

http://www.hindawi.com
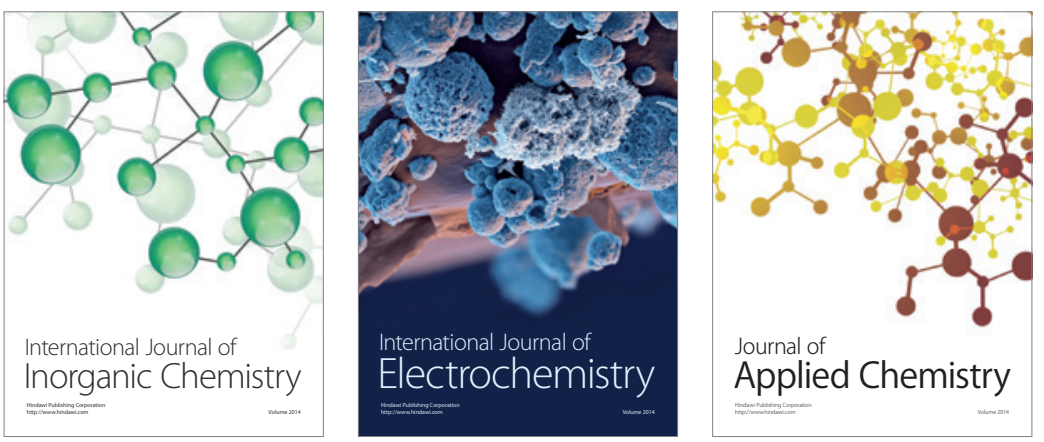

Journal of

Applied Chemistry
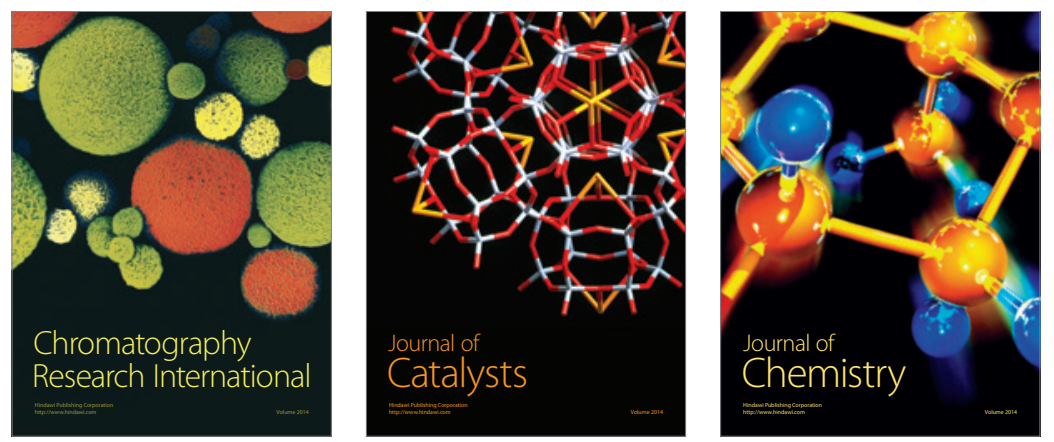
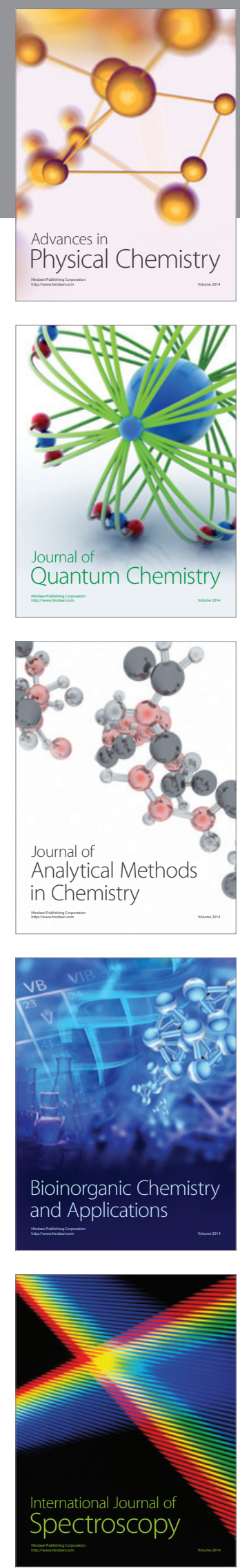